\title{
Leveraging the Fulcrum Point in Robotic Minimally Invasive Surgery
}

\author{
Caspar Gruijthuijsen, Lin Dong, Guillaume Morel, and Emmanuel Vander Poorten
}

\begin{abstract}
In robotic Minimally Invasive Surgery (MIS) the incision point acts as a fulcrum around which the surgical instrument pivots. The fulcrum point has been the topic of much research. Mechanisms have been invented to enforce instrument motion about such a fulcrum. Other systems establish a fulcrum through coordinated control of their joints. For laparoscopists, the fulcrum point is an obstacle to overcome through a lot of training. For robots, it is a hurdle requiring careful consideration.

In this paper, new estimation methods are proposed to exploit the properties of a fulcrum and turn its presence into an advantage. The paper starts by presenting a novel fulcrum estimation method that is robust against measurement noise and quantization effects. A general fulcrum refinement method is proposed next. This method can be used as add-on to ameliorate alternative estimation approaches. It is shown how the fulcrum can also be leveraged to get accurate, high-bandwidth estimates of the instrument tip. The quality with which the instrument tip is estimated has a large impact on the performance of advanced guidance schemes, such as haptic virtual walls. User tests are included, demonstrating substantially improved guidance thanks to the algorithms presented in this work.
\end{abstract}

Index Terms-Medical robots and systems, surgical robotics: laparoscopy, haptics and haptic interfaces

\section{INTRODUCTION}

$\mathbf{F}$ OR many surgical interventions Minimally Invasive Surgery (MIS) is becoming the standard. In MIS, surgical instruments pass through small incisions in the patient's skin. Smaller incisions lead to smaller scars, but smaller incisions also constrain the instrument motion degrees of freedom (DoFs) to a greater extent. The instrument can pivot about the incision point; it can rotate about and translate along its longitudinal axis, but motion is constrained in the directions tangential to the body wall. The incision thus acts as a fulcrum which introduces other difficulties for the surgeon. Motion and forces are inverted and scaled. The scale factor varies with insertion depth. This variable scaling complicates correct perception of the forces that are exerted at the instrument tip. Friction forces that develop between the instrument and the incision and torques that are required to deform the body wall,

Manuscript received September, 10, 2017; Revised Januari, 3, 2018; Accepted Februari, 8, 2018.

This paper was recommended for publication by Editor-in-Chief A. Bicchi and Editor K. Masamune upon evaluation of the Reviewers' comments. This work was supported by an FWO travel grant and by internally funded projects at KU Leuven (PRESLICE-3E160397 and STABLEYES-3E160419).

C. Gruijthuijsen and E. Vander Poorten are with the Department of Mechanical Engineering, KU Leuven, 3000 Leuven, Belgium firstname. lastnameekuleuven.be

L. Dong and G. Morel are with ISIR, Sorbonne Universités UPMC Univ. Paris 06, UMR 7222, Paris, France lastnamedisir.umpc.fr

Digital Object Identifier (DOI): see top of this page. e.g. to incline the instrument up to a certain angle, further mask the haptic perception.

A key motivation for introducing robotic surgery in the ' $80 \mathrm{~s}$ consisted exactly in bypassing problems of motion inversion and variable scaling associated to the presence of the fulcrum. Knowledge of the fulcrum location was incorporated in the robot navigation and control algorithms. By doing so, the complex instrument handling from MIS could be transformed into a simpler tracking and positioning problem in Cartesian space. Surgeons were relieved as this resembles the situation of instrument handling in open surgery more closely.

Rigid link MIS robots can be roughly classified in four categories based on the way they deal with the fulcrum point. In the first category, robots possess a fixed pivoting point in their workspace, by their mechanical design. This point, the Remote Center of Motion (RCM), lies outside of the robot's mechanism. Before starting the intervention, the robot is servoed so as to maximally align the RCM with the incision. The second category comprises the so-called Local Center of Motion (LCM) systems. Systems such as the body-mounted Ligth Endoscope Robot (LER) [1], its successor VikY [2] and the $\mathrm{MC}^{2} \mathrm{E}$ robot [3] are representative for this category. LCM systems possess a 'local' fulcrum point that is an integral part of their linkage structure and that is made to coincide with the patient's incision. Robots with a 'virtual' RCM form the third category. These robots have no particular mechanisms that constrain their motion, but they are programmed to maintain a 'virtual' fulcrum point through coordinated control of a plurality of their joints. Robots of this category may have e.g. an anthropomorphic structure, like DLR's MIRO robot [4]. Finally, a fourth category consists of 'passive' wrist robots. At the end of their kinematic chain, these robots typically possess a spherical wrist, to which the instrument is hinged. The axial rotation of the instrument is controlled, but the direction of the instrument axis is not constrained. Therefore, the instrument will freely pivot around the constraining point, namely the incision point in the patient. A passive wrist is thus always aligned with the incision.

Accurate knowledge of the incision point is important for all these robotic MIS approaches. For the mechanical RCM and LCM approaches, the location of the incision point needs to be accurately aligned with the center of motion [5] of the mechanism. Improper alignment may lead to excessive forces on both instrument and patient. An increased prevalence of Trocar Site Herniation (TSH) after robotic surgery has been reported [6], [7]. In virtual RCM approaches, the position of the incision must be known and expressed w.r.t. the robot base frame, in order for the software to be able to enforce a fulcrum 
constraint [8], [9], [10], [11]. Inaccuracies may cause harm to or lead to dangerous situations for the patient.

In passive wrist robots, contrarily, misalignment does not lead to excessive forces on the body wall. By their nature, the incision constraint cannot be violated and as such, these robots are considered safer than virtual RCM approaches. They are also easier to set up than mechanical RCM approaches, which require an alignment procedure. Still, knowledge of the fulcrum point is crucial for their control. In fact, this knowledge lies at the genesis of passive wrist robots: by virtue of it, wrist actuators can be eliminated and the mechanical construction greatly simplified [12]. However, this reasoning implies that inaccuracies in the fulcrum position will also lead to inaccuracies in the instrument control. As such, for passive wrist robots, an identification step is also needed to accurately locate the incision.

Since the instrument tip position depends on the location of the incision, inaccurate knowledge of the latter translates to incorrect estimates of the tip position. Whereas during set up of MIS robots of the first three categories an alignment procedure is required to avoid TSH, for passive RCM robots an identification step is needed to precisely quantify the location of the incision.

The above illustrates the importance of accurate knowledge of the fulcrum position in robotic MIS. This paper introduces a new, more robust method to estimate the location of the fulcrum point. Additionally, a method is proposed that exploits the estimated fulcrum position to improve the pose estimation of the surgical robot itself. Moreover, it is shown how specific characteristics of the fulcrum point can be exploited to improve the stability of some haptic control schemes. This method can also be applied to any fulcrum estimation approach found in literature. In this paper, the presented work is principally applied to passive wrist robots, but it is also more generally applicable.

The structure of the paper is as follows. In Sec. II, methods for fulcrum estimation will be discussed. A new method for fulcrum estimation that can cope with high measurement noise is proposed in Sec. III. Next, Sec. IV shows how to improve the tip position measurement. A series of simulations and invitro experiments are described in Sec. V, showing the benefits of the proposed methods. After a discussion of the obtained results in Sec. VI, the paper is concluded in Sec. VII.

\section{STATE OF THE ART}

This section surveys a number of methods for fulcrum estimation that have been proposed in literature. The methods can be largely classified in three groups: methods that rely on (1) external measurement equipment, (2) force sensors, or (3) kinematic data only. Depending on the application, some of these works target RCM alignment, others target robot control, or both. Yet other works estimate the fulcrum position to help real-time instrument tracking in endoscopic images [13], [14]. Indeed, the fulcrum location can help generate seed points for more accurate initialization of instrument segmentation routines.

\section{A. Fulcrum estimation by external measurement equipment}

Several approaches have been developed where the fulcrum is estimated using measurement equipment that is set up externally w.r.t. the surgical robot. Mostly, this concerns cameras positioned within the line of sight. These methods try to detect instrument axes within the generated images. Typically, least squares or other optimization methods are employed to calculate the best intersection between the detected lines. The approaches mainly differ in the methods adopted to detect edges in the image. Some methods express the results in the image space, others in the Cartesian space. Also the type of employed camera may differ.

Krupa et al. make use of optical trackers attached to the instrument shaft to detect the instrument [15]. While assuming a motionless fulcrum, the intersection between two detected axes is computed, leading to a fulcrum estimate expressed in image space. Nageotte et al. study a number of approaches to recover the 3D fulcrum position from monocular images [16]. They start from the assumption that an edge detection algorithm has already located the instrument borders in the images, with possible errors. Then, they present three methods and a refinement strategy to estimate the fulcrum, mostly based on perspective calculus. Their study elaborates on the optimal conditions for each of their approaches. Wilson et al. present a stereocamera approach [17]. First, edge detection is performed via user interaction. Then, the instrument's centerline is computed in the images of the mutually calibrated cameras. Via triangulation, a 3D representation is obtained. The intersection of the $3 \mathrm{D}$ centerlines is treated as the fulcrum location. Alternatively, mutually registered images can be captured by moving a robot-mounted camera over different poses. Voros et al. ask the surgeon to identify the fulcrum point in two registered images via a voice-controlled mouse, after which the 3D position is computed [14]. Rosa et al. ask the operator to carefully pivot a calibration instrument through the incision. The Hough method and pruning methods are applied to automatically detect the instrument in the large set of stereocamera images. A least squares fit is adopted to compute the 3D fulcrum point [5]. The fulcrum position - or incision in the patient - may also be planned during a preoperative phase. Navigation systems that help register the patient to the pre-operative plan can then be used to assist the surgeon in making the incision at the correct location [9], [18].

Most above approaches include some procedural steps that involve supervision of an operator. This makes it somewhat cumbersome for use in clinical applications. Furthermore, an additional registration step is typically needed in order to express the estimated fulcrum position in the robot space. Registration is hard to automate and serves as a source for potential errors. These arguments indicate other approaches might be better suited for automatically obtaining the fulcrum in the robot space.

\section{B. Fulcrum estimation from force sensing}

Since the body wall has a non-negligible thickness, there will always be some forces (and torques) generated as the instrument is pivoting about the incision. The larger the 
misalignment between the RCM and the incision, the larger the generated forces will be. By measuring these forces, e.g. via a dedicated force/torque sensor, it is thus possible to estimate the location of the fulcrum point. Krupa et al. propose to apply a reference excitation force signal to estimate the tissue stiffness at the incision point. From this, they derive the fulcrum location [12]. It is also possible to estimate the location of the fulcrum by measuring the induced forces when pivoting the instrument about a known point along its axis and by exploiting the lever principle [19], [20].

When the force sensor is mounted on the robot, these approaches offer an estimate that is directly expressed in the robot space, thus eliminating the need for an additional registration procedure. However, most methods require that the instrument is only contacting at the incision point and thus not at the tip. The aforementioned works build upon the assumption that the incision point exerts a pure force on the instrument. This ignores the fact that significant torques are applied on the body wall, just to be able to incline the instrument [21], even when the system is perfectly aligned. Force sensors are noisy and prone to drift. They require calibration, pose difficulties when it comes to sterilization and are expensive. Also, the position of the force sensor must be known for fulcrum estimation, e.g. by mounting them on the surgical robot and using the robot kinematics.

\section{Fulcrum estimation from kinematic data}

For robots that do not have a fixed center of motion, the robot's kinematic data can be exploited to estimate the fulcrum position. Obviously, kinematic data is readily available and fulcrum estimates will be conveniently expressed in robot space. While ideally measurement data should reflect that the instrument passes through a single fixed point in space, in practice this is not the case, due to measurement noise, motions of and forces applied to the incision point. Similarly to camerabased approaches, kinematic approaches locate the incision by searching for the intersection that minimizes the distance to all recorded instrument axes.

An early approach proposed to record two sequential axes. The point in space where the mutual distance between the axes is minimal, was considered as fulcrum. Ortmaier et al. repeated this process for multiple axis pairs and averaged the resulting points [22]. Dong et al. generalized this method by forming it into a least squares estimation problem [23]. Each time the instrument has sufficiently moved, the pose of the axis is added to a circular buffer of size $n$. The best intersection between the axes in the buffer yields the fulcrum estimate.

Dong's method is appealing, as it allows detecting in parallel the fulcrum's presence ( $\operatorname{small} n$ ) and its position (large $n$ ). It also updates the fulcrum when the incision moves. By appropriate choice of $n$, a trade-off can be made between accuracy and mobility of the fulcrum estimate. However, the method does not fully incorporate the fact that the position and orientation are often decoupled in MIS robots. For instance spherical wrists, commonly employed in virtual RCM or passive wrist systems, noise on orientation sensors will have a large impact on the estimated fulcrum location. Fig. 1

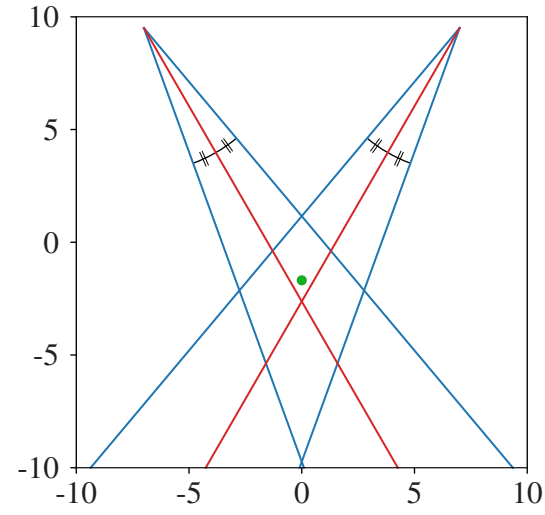

Figure 1: Least squares solution for best line intersection (green) between two noiseless measurements (red) and four synthetic, 'noisy' measurements at equal angles (blue). The estimated intersection differs from the ground truth at the intersection of the red lines.

illustrates how methods based on least squares estimates will systematically misjudge the incision depth in case of noisy orientation measurements. It depicts, for a simplified case with two correct measurements and four synthetic, 'noisy' measurements, how the fulcrum estimate is systematically pulled towards the robot's wrist.

The next section introduces an algorithm that can robustly handle noisy situations.

\section{EXTENDED KALMAN FILTER FOR FULCRUM ESTIMATION}

An Extended Kalman Filter (EKF) was developed for estimating the fulcrum point, based on kinematic data of the instrument. This method is tailored for cases where the available data consists of instrument poses measured by a robotic manipulator with a decoupled position and orientation measurement. For other robot topologies, where this condition is not fulfilled, the method can be straighforwardly expanded by incorporating the robot kinematic model in the measurement model of the EKF.

The governing equations for the EKF are given as [24]:

$$
\begin{aligned}
\mathbf{x}_{k} & =\mathbf{g}\left(\mathbf{x}_{k-1}\right)+\boldsymbol{\varepsilon}_{k}, \\
\mathbf{z}_{k} & =\mathbf{h}\left(\mathbf{x}_{k}\right)+\boldsymbol{\delta}_{k},
\end{aligned}
$$

with $\mathbf{x}_{k}$ the current state vector and $\mathbf{x}_{k-1}$ the state vector at the previous estimation step. The prediction model is described by the function $\mathbf{g}()$. $\boldsymbol{\varepsilon}_{k}$ expresses the noise on the prediction. Further, $\mathbf{z}_{k}$ is the measurement vector at time $k, \mathbf{h}()$ the measurement model and $\boldsymbol{\delta}_{k}$ the measurement noise vector.

The state vector is defined as:

$$
\mathbf{x}_{k}=\left[\begin{array}{llllll}
p_{x, k} & p_{y, k} & p_{z, k} & f_{x, k} & f_{y, k} & f_{z, k}
\end{array}\right]^{T},
$$

with $\mathbf{p}=\left[\begin{array}{lll}p_{x} & p_{y} & p_{z}\end{array}\right]^{T}$ the position of the end effector and $\mathbf{f}=\left[\begin{array}{lll}f_{x} & f_{y} & f_{z}\end{array}\right]^{T}$ the position of the fulcrum (Fig. 2.a).

For the prediction step a very simple motion model can be used: 


$$
\mathbf{x}_{k}=\mathbf{g}\left(\mathbf{x}_{k-1}\right)+\boldsymbol{\varepsilon}_{k}=\mathbf{x}_{k-1}+\boldsymbol{\varepsilon}_{k} .
$$

This model assumes a constant position for $\mathbf{p}$ and $\mathbf{f}$. For $\mathbf{p}$, the noise component in $\boldsymbol{\varepsilon}$ is relatively large, while for $\mathbf{f}$ it is smaller, depending on the desired sensitivity to motion of the fulcrum.

For the measurement model, as argued above, it is assumed that the robot can measure the pose of the instrument w.r.t. its base frame. As the rotation of the instrument about its axis is irrelevant for fulcrum estimation, it suffices to measure the instrument's direction vector $\mathbf{i}$, represented with an altitudeazimuth angle pair $(\alpha, \beta)$ :

$$
\mathbf{i}=\left[\begin{array}{lll}
i_{x} & i_{y} & i_{z}
\end{array}\right]^{T}=\left[\begin{array}{lll}
\cos \alpha \cos \beta & \cos \alpha \sin \beta & \sin \alpha
\end{array}\right]^{T},
$$

The end effector position is measured directly. This allows to summarize the measured data as:

$$
\mathbf{z}_{k}=\left[\begin{array}{lllll}
\tilde{p}_{x, k} & \tilde{p}_{y, k} & \tilde{p}_{z, k} & \tilde{\alpha}_{k} & \tilde{\beta}_{k}
\end{array}\right]^{T} .
$$

A tilde is used to notify that it concerns measured data. Considering that the instrument position and orientation information are contained in the state vector, and after introducing the auxiliary variable $\mathbf{d}=\mathbf{f}-\mathbf{p}=\left[\begin{array}{lll}d_{x} & d_{y} & d_{z}\end{array}\right]^{T}$, the measurement model yields:

$$
\mathbf{z}_{k}=\mathbf{h}\left(\mathbf{x}_{k}\right)+\boldsymbol{\delta}_{k}=\left[\begin{array}{c}
p_{x, k} \\
p_{y, k} \\
p_{z, k} \\
\operatorname{atan} 2\left(d_{z, k}, \sqrt{d_{x, k}^{2}+d_{y, k}^{2}}\right) \\
\operatorname{atan} 2\left(d_{y, k}, d_{x, k}\right)
\end{array}\right]+\boldsymbol{\delta}_{k} .
$$

The components of the measurement noise $\boldsymbol{\delta}$ have to be set in accordance with the specific hardware. To obtain directionindependent orientation noise, the noise on $\alpha$ and $\beta$ may be modelled as $\mathcal{N}\left(0, \sigma_{\alpha}^{2}\right)$ and $\mathcal{N}\left(0, \frac{\sigma_{\alpha}^{2}}{\cos ^{2} \alpha}\right)$, respectively, because

$$
\left\|\frac{\partial \mathbf{i}}{\partial \alpha}\right\|=1 \quad \text { and } \quad\left\|\frac{\partial \mathbf{i}}{\partial \beta}\right\|=\cos \alpha .
$$

It is important to note that discontinuities can occur in $\beta$ when the EKF algorithm internally subtracts the actual measurement from the evaluation of the measurement model and the $\beta$-terms are on opposite ends of $[-\pi, \pi[$-interval.
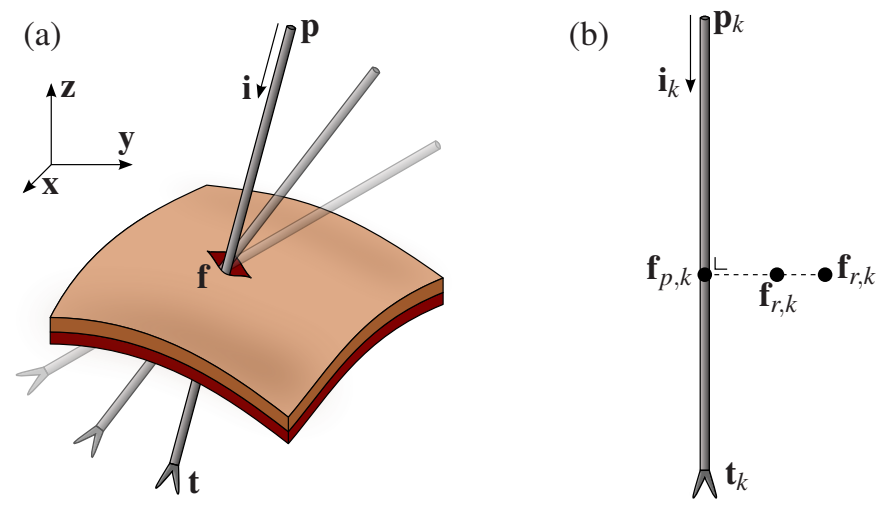

Figure 2: Representation of the symbols used for (a) the EKF and (b) the fulcrum refinement.
These jumps can be avoided by normalizing the subtraction result to the $[-\pi, \pi[$-range.

With the above equations, the EKF is completely specified. As the state vector contains the fulcrum position, its estimate will naturally emerge from the EKF algorithm at runtime, with the certainty of the estimate increasing over time.

\section{FULCRUM REFINEMENT FOR TIP POSITION MEASUREMENT}

Measuring the position of the tip of a surgical instrument can be a delicate issue. In MIS, long and slender instruments are attached to the robot wrist. Therefore, the accuracy with which the tip position can be estimated depends to a large extend on the accuracy with which the wrist orientation is measured. As in any real-world application, noise and inaccuracies have to be carefully handled. Considering passive wrist robots, they typically have no or very low transmissions in their joints, as they need to be back-drivable. The low reduction ratio does not allow raising the measurement resolution and precision. Also, for other robots, the wrist sensors have comparatively low quality, because of e.g. space considerations. Given that any orientation measurement error is amplified by the instrument length, accurate measurement of the robot tip solely relying on robot proprioception is difficult. In the following, a new approach is introduced, which makes use of the estimated fulcrum point to enhance the tip position measurement. In Sec.V-D, it will be shown how this not only contributes to an improved quality of tip measurement, but also helps to reach better haptic performance.

A first approach one might consider in order to remove orientation measurement noise, would be to apply a low pass filter on the orientation data. However, real-time filtering requires a causal filter. The unavoidable phase lag would limit the accuracy of tip position estimates, and hence it would also be detrimental for the performance of any controller that uses this data. A second approach that could be considered, consists of drawing a line between the wrist center and through the fulcrum point. From the knowledge of the instrument length, it is then possible to determine the tip position. This method does not introduce phase lag: measurements of the wrist position produce a tip measurement at the same rate. However, the method relies on good knowledge of the fulcrum position. As there will be inaccuracies in fulcrum estimation, also due to the fact that the incision may move over time, its estimate may be inaccurate. Especially when the wrist comes close to the fulcrum, errors in the estimated fulcrum location are strongly amplified towards the tip.

In summary, the precision with which the tip position can be estimated from raw wrist orientation measurements is poor, but the accuracy can be good in the sense that there are no systematic errors. By filtering the orientation estimate can be improved at the cost of phase lag. The tip position can be computed at high bandwidth and with great precision from the fulcrum estimate, but it has poor accuracy, e.g. when the estimated fulcrum position deviates from the actual one. Note that a deviation can be detected when observing that the estimated fulcrum position does not coincide with the measured 
instrument axis. This is because in reality the instrument axis and the incision are to coincide as long as the instrument is inserted in the body.

In the following, a method is introduced that uses the best of all worlds: the accuracy of the raw orientation measurements, the noise rejection of the filtering, and the delay-free high bandwidth thanks to the fulcrum estimate. While filtering the wrist orientation induces a delay, filtering the fulcrum position forms less of a problem, as it is assumed that this position is (approximately) stationary ${ }^{1}$. To this end, consider the fulcrum estimate $\mathbf{f}$, the wrist position $\mathbf{p}$ and the measured direction vector of the instrument $\mathbf{i}$. The projection of $\mathbf{f}$ onto the instrument axis is formulated as:

$$
\mathbf{f}_{p}=\mathbf{p}+[(\mathbf{f}-\mathbf{p}) \cdot \mathbf{i}] \mathbf{i} .
$$

As $\mathbf{f}_{p}$ is projected directly on the instrument axis, it is noisy and not usable for stable tip estimation. Instead, $\mathbf{f}$ can be set to track $\mathbf{f}_{p}$ with a low correction gain $\gamma$. This yields a 'refined' fulcrum estimate $\mathbf{f}_{r}$ (Fig. 2.b):

$$
\mathbf{f}_{r, k}=\mathbf{f}_{r, k-1}+\gamma\left(\mathbf{f}_{p, k}-\mathbf{f}_{r, k-1}\right),
$$

with $k$ the time step. This is equivalent to applying an exponential filter to the projected fulcrum:

$$
\mathbf{f}_{r, k}=(1-\gamma) \mathbf{f}_{r, k-1}+\gamma \mathbf{f}_{p, k} .
$$

With a proper, close to zero, choice of $\gamma$, it is ensured that $\mathbf{f}_{r}$ will move towards the instrument axis. Using $\mathbf{f}_{r}$ and instrument length $l$, the tip position estimate $\mathbf{t}$ can be obtained as:

$$
\mathbf{t}=\mathbf{p}+\frac{\mathbf{f}_{r}-\mathbf{p}}{\left\|\mathbf{f}_{r}-\mathbf{p}\right\|} l .
$$

With this new estimate for the tip position, systematic errors, such as those arising from an off-axis fulcrum estimate, are eliminated. Noise on $\mathbf{t}$ is suppressed thanks to the exponential filter. The measurements have no phase lag w.r.t. p. The proposed method can be used in combination with any fulcrum estimation approach.

\section{EXPERIMENTS}

The proposed algorithms are validated in this section. The fulcrum estimation and the tip position measurement methods were validated both in simulation and experimentally. Each time, the outcome is compared to the least squares method by Dong et al. [23], which is representative for the current state-of-the-art.

\section{A. Description of the simulation experiment}

In this subsection the details of the simulations that were conducted are summarized. To assess the robustness and the responsiveness to measurement noise of the fulcrum estimation methods, the behaviour of a robotic manipulator holding an instrument and constrained by an incision into a patient, was simulated. The topology of the robot was such that the position

\footnotetext{
${ }^{1}$ In a future extension outside the scope of this work, this assumption may be relaxed by superposition of an estimated trajectory that e.g. reflects the periodic motion caused by breathing or other physiological phenomena.
}

and the orientation of the end effector were decoupled, by means of a spherical wrist. The kinematics of the simulated robot were based on Achilles, which will be detailed in a Sec. V-D. The simulation environment was built up so that it was straightforward to introduce both Gaussian and quantization noise on the orientation measurements in the wrist. The position of the wrist was assumed to be perfectly known. Since this concerns a simulation setup, the ground truth for the fulcrum position is available and a deviation from this ground truth can be easily analysed. For each simulation experiment, the robot end effector was programmed to move over a circular trajectory with a diameter of $15 \mathrm{~cm}$ at a height of $25 \mathrm{~cm}$ above the fulcrum. Each revolution took $2 \mathrm{~s}$ and the simulation time step was $1 \mathrm{~ms}$.

\section{B. Robustness of fulcrum estimation against measurement noise}

To estimate the robustness of the fulcrum estimation method against measurement noise, the circular trajectory was applied, while measurement noise was added to the orientation measurements. During this motion, the fulcrum was estimated by Dong's method, with varying buffer size (respectively 10, 20 and 100 samples), and with the EKF method. The simulation was run as long as needed to fill all buffers. The fulcrum error was characterised by the Cartesian distance between the ground truth and the estimated fulcrum position. Fig. 3.a and Fig. 3.b show the fulcrum error when respectively Gaussian noise with an increasing standard deviation and quantization noise with an increasing quantization step (decreasing resolution) were added. All methods handle low noise very well, but it can clearly be seen that the EKF method is more robust against Gaussian noise than the state-of-the-art method. By increasing the buffer size, latter method does keep its performance over a wider noise range. For quantization noise the EKF still outperforms the state-of-the-art method, but the effect is less pronounced. As the Gaussian noise increases, the state-of-the-art method converges towards a systematic error. Basically, the solution converges towards the center of the circular trajectory of the end effector, in this case $25 \mathrm{~cm}$ above the fulcrum. This corresponds to the effect mentioned in Fig. 1.

\section{Responsiveness of fulcrum estimation}

The simulation was conducted once more to assess the responsiveness of the fulcrum estimation methods. The robot end effector was made to move once more over the aforementioned circular trajectory. Between $10 \mathrm{~s}$ and $13 \mathrm{~s}$ the instrument was held stationary. During this period the fulcrum was then displaced $5 \mathrm{~cm}$ sideways. Gaussian noise with a standard deviation of $0.2^{\circ}$ was added to the orientation measurements. Dong's method, with varying buffer sizes, and the EKF method were run for fulcrum estimation. Fig. 3.c, and its close-up in Fig. 3.d, show how the fulcrum error evolves over time during the described motions. It can be seen that at start-up the EKF method responds immediately but converges a bit slower than Dong's method with a small buffer. However, when the fulcrum moves, the EKF method always keeps on tracking the instrument axis. This explains why for the EKF the fulcrum 
(a)

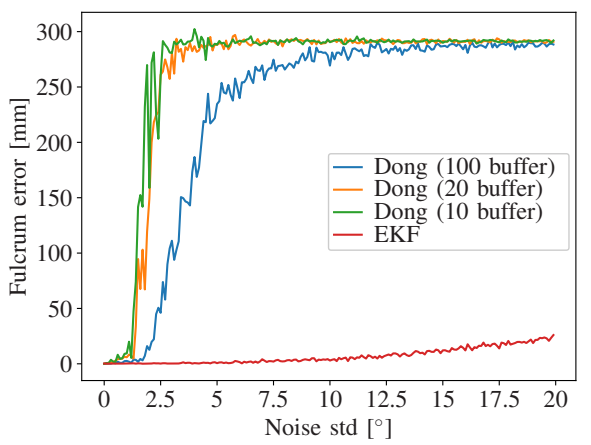

(b)

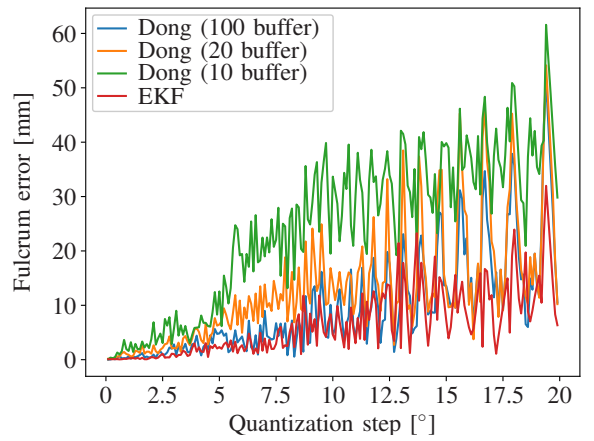

(c)

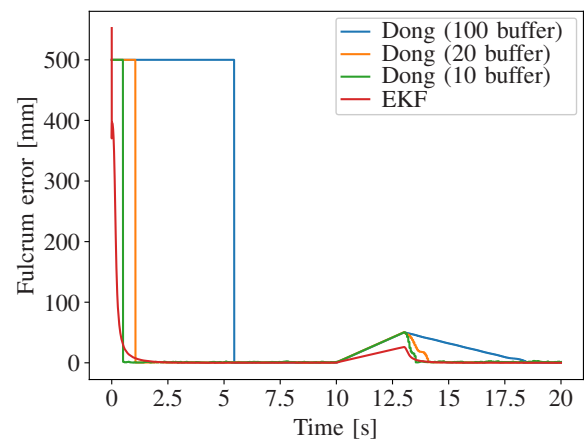

(d)

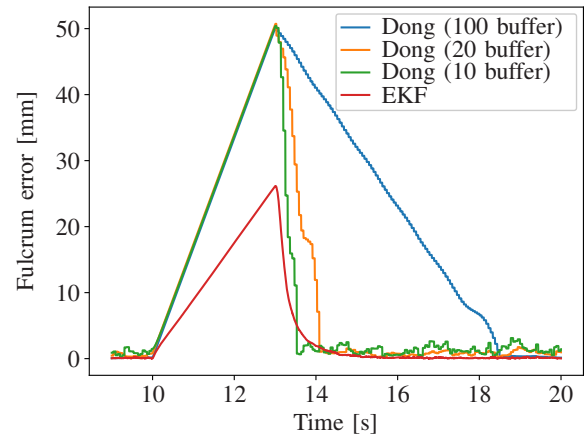

(e)

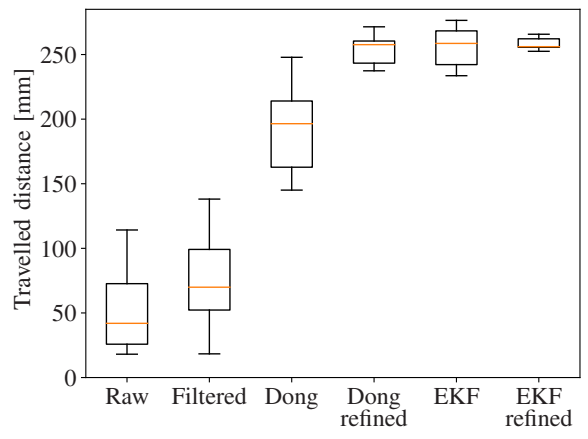

(f)

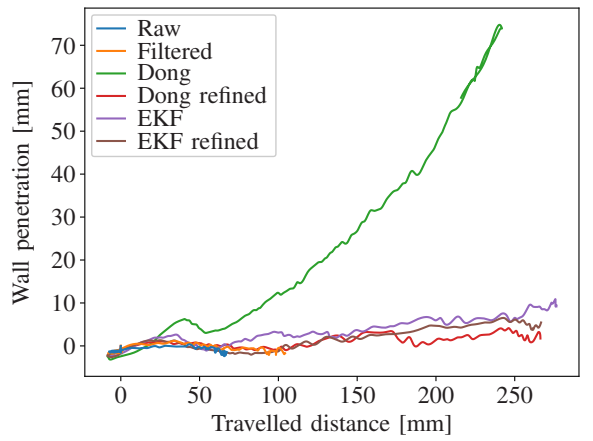

Figure 3: Experimental results. The simulated fulcrum error is presented for different fulcrum estimation approaches, (a) with increasing Gaussian noise on the instrument orientation measurement, (b) with increasing quantization noise on the instrument orientation measurement, and (c) as a function of time, with an external fulcrum motion at $10-13 \mathrm{~s}$. A close-up of (c) around the time of the fulcrum motion is shown in (d). The results for haptic tip control with different tip measurement approaches are shown in (e)-(f). The box plots in (e) illustrate for each tip measurement approach how far the instrument tip had travelled in contact with the virtual wall, at the moment test subjects ended the experimental run, because of unsafe wall behaviour or task completion. (f) indicates how the effective wall penetration (positive $=$ no penetration) evolved in function of the travelled distance.

error never reaches a $5 \mathrm{~cm}$ error. Contrarily, the state-of-the-art method only starts compensating for the fulcrum motion when the effector resumes its circular trajectory at $13 \mathrm{~s}$, as motion is necessary to update the buffer and thus excite the algorithm.

\section{Haptic tip control}

To evaluate the value of the newly proposed instrument tip estimation method (that also relies on fulcrum estimation), a user experiment was set up. Achilles, a passive wrist robot, which is a modified version of the Virtuose3D (Haption SA, Laval, France), was used to this end. Achilles' controller runs at $1 \mathrm{kHz}$, which is suitable for haptic control. The wrist position is measured with highly precise encoders offering a position resolution in the $\mu \mathrm{m}$-range. The wrist orientation is measured with potentiometers. The noise on these was found to have a standard deviation of $0.2^{\circ}$. A $40 \mathrm{~cm}$ instrument was attached to Achilles' wrist. For the experiment the instrument was inserted through a fixed, mechanical fulcrum (Fig. 4).

Test subjects were asked to comanipulate the instruments together with the robot, such that the instrument tip slides over a virtual wall, which was placed horizontally at a distance of $7.5 \mathrm{~cm}$ below the fulcrum. A virtual wall with a stiffness of $2000 \mathrm{~N} / \mathrm{m}$ was implemented in the robot controller. The wall is representative for a forbidden region that may be programmed in an MIS scenario to protect fragile areas. The users are asked to start with the instrument oriented perpendicularly w.r.t. the wall. They are to slide the instrument sideways, while keeping soft contact with the virtual wall. To be able to follow the wall, the users need to insert the instrument gradually deeper through the fulcrum. Fig. 4 illustrates how this gesture takes place. As the instrument moves sideways and deeper, its axis becomes more parallel to the wall. This represents an increasingly difficult situation for the virtual wall controller. Indeed, correct estimation of the orientation becomes increasingly more important to determine the penetration of the tip in the virtual wall. Also, due to fulcrum effect [25], the stiffness that needs to be rendered at the wrist rises. The subjects were asked to stop sliding over the wall when the instrument was fully inserted, or when they considered it too unsafe to proceed under a clinical condition, e.g. when overly large vibrations occurred.

For this experiment six different approaches to estimate the tip position were implemented and used by the virtual wall controller. The tip position was calculated 1) using the wrist position in combination with the raw wrist orientation measurement and 2) with a filtered wrist orientation measurement (cut-off frequency at $10 \mathrm{~Hz}$ ), 3) using the wrist position 


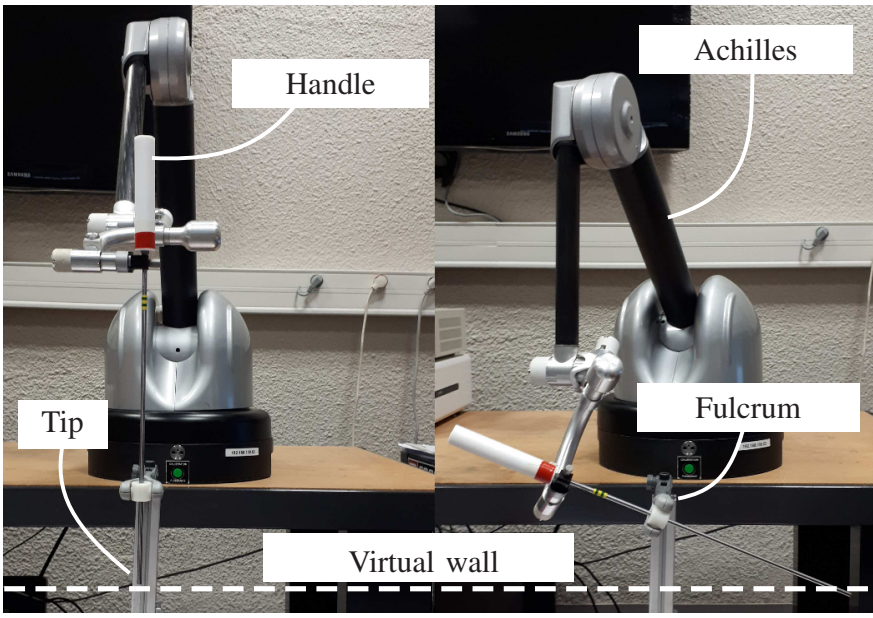

Figure 4: The experimental setup for haptic tip control. Left: the initial condition, with the instrument inserted through the fulcrum and touching the virtual wall perpendicularly. Right: the situation after the instrument tip has travelled some distance over the virtual wall.

in combination with a fulcrum estimate based on Dong's method, 4) based on Dong's method, plus the additional fulcrum refinement step from Sec. IV, 5) based on the EKF, and 6) based on the EKF, plus the fulcrum refinement step. Five subjects were asked to do the task twice with all six tip measurement approaches. The methods were presented in a random order to the test subjects.

Fig. 3.e shows box plots of the distances that subjects were able to slide the instrument tip over the virtual wall. The figure shows that estimating the tip via the fulcrum position yields better haptic control. Subjects felt confident and safe in regions that were far more challenging for the haptic controller. Fig. 3.f shows the actual wall penetration of the instrument tip. To obtain the actual tip position, the raw position and orientation data were post-processed and filtered by a noncausal, zero-phase filter. The wall penetration is plotted in function of the distance travelled over the wall. Most methods yield a wall penetration of around zero. This corresponds to the expectations, as the instrument tip is supposed to softly touch the wall. While users were able to travel further over the wall with Dong's method, compared to raw or filtered estimates, it turns out that the actual tip position drifts away from the location of the wall. This phenomenon arises because the method from Dong does not ensure that the fulcrum estimate is on the instrument axis. As can be seen, such deviation can cause large, systematic errors towards the tip, as earlier argued in Sec. IV. For the other three methods the fulcrum estimate is attracted towards the instrument axis and as such does not suffer from this phenomenon.

\section{DISCUSSION}

From the experiments, it can be seen that the EKF has a number of advantages over state-of-the-art methods that are typically based on least squares estimation.
Firstly, as visible in Fig. 3.a, the EKF-based method is more robust against measurement noise. In a surgical situation robustness is vitally important. Even when sensors with lower performance are used, fairly accurate estimates of the fulcrum can still be obtained. Also in the presence of quantization noise the performance of the EKF outperforms other methods, although to a lesser extent (see Fig. 3.b).

Secondly, the method does not require a minimal amount of excitation, i.e. change in instrument pose, before updating its estimate. Other methods do not update the fulcrum's estimate when the instrument is kept stationary. Contrarily, the EKF method pulls the fulcrum estimate towards the instrument axis, even if the latter shows no motion. This behaviour is desired, as in a normal situation the instrument will always pass through the fulcrum.

Thirdly, because a minimum excitation is not needed, each measurement at $1 \mathrm{kHz}$ is taken into account. As such, the EKF method is able to update the fulcrum estimate more quickly than other methods (Fig. 3.c-3.d). The advantage here is twofold. In the initialization phase of the algorithm, it takes less time and less probing around with the inserted instrument to find a good fulcrum estimate. This makes that the initialization procedure becomes less cumbersome and also more fail-safe. Analogously, the EKF method can quickly accommodate in case the fulcrum moves. Patient movement or movement of the fulcrum due to physiological phenomena or tissue deformation cannot be excluded. The sensitivity of the filter to motion of the fulcrum can be easily tuned to fit to a particular situation by adjusting the covariance in the prediction function for the fulcrum position.

Thanks to the experiments, the value of the EKF's property of attracting the fulcrum estimate towards the instrument was recognized. The fulcrum refinement method from Sec. IV can be added to transfer this behaviour also to other fulcrum estimation approaches that lack such property. Fig. 3.f shows how Dong's method experiences problems to get an accurate tip measurement. However, after application of the fulcrum refinement method, the error in Dong's method vanishes too.

Finally, based on the box plots in Fig. 3.e one understands that tip estimates that rely on estimated fulcrum positions also improve the stability of the haptic controller. This is especially true when dealing with a robot that has noisy orientation sensors in its wrist. This is a common condition when for instance direct drive, passive wrist robots are concerned. Indeed, both [22] and [26] indicate that noise jeopardized their controller stability.

Note that shaft flexion can jeopardize the estimation of the fulcrum position as well as the instrument tip position. When small diameter instruments are used, this problem is more prominent, as this goes unnoticed by proximal pose measurements. If flexion occurs temporarily, the fulcrum estimation can be robustified by increasing the EKF measurement noise $\boldsymbol{\delta}$ related to i. Otherwise, additional sensing, such as force sensing, may be needed to improve the position estimates.

\section{CONCLUSION}

This papar studies the fulcrum point in robotic MIS. An EKF-based method for fulcrum estimation was introduced. 
This method was particularly interesting for its noise rejection capabilities. Additional advantages of this method, mainly for real-time applications, are its responsiveness to changes in the fulcrum position and its property to attract the fulcrum estimate towards the instrument axis, even when the instrument is stationary. Other fulcrum estimation approaches from literature do not take into account that the fulcrum estimate should always coincide with the instruments axis in MIS. A fulcrum refinement strategy has been proposed that allows upgrading prior works to incorporate this knowledge. It is demonstrated in this work that accurate knowledge of the fulcrum position provides a means to obtain accurate and highbandwidth estimates of the tip position. As the method avoids measurement delays and does not introduce the systematic errors other methods do, it is of particular use for building haptic control schemes. The paper showed how this improved tip position estimation allows a substantial enhancement of haptic guidance schemes. All above methods focused on passive RCM robots, but are thought to be more broadly applicable. A validation of the presented methods in an invivo setting remains the topic of future work.

\section{ACKNOWLEDGMENT}

The authors want to thank Haption for the support. Haption SA (Laval, France) is a manufacturer of hardware and software solutions for force feedback.

\section{REFERENCES}

[1] P. Berkelman, E. Boidard, P. Cinquin, and J. Troccaz, "Ler: the light endoscope robot," in 2003 IEEE/RSJ International Conference on Intelligent Robots and Systems, vol. 3, 2003, pp. 2835-2840.

[2] S. Voros, G. P. Haber, J. F. Menudet, J. A. Long, and P. Cinquin, "Viky robotic scope holder: Initial clinical experience and preliminary results using instrument tracking," IEEE/ASME Trans. Mechatron., vol. 15, no. 6, pp. 879-886, 2010.

[3] N. Zemiti, G. Morel, T. Ortmaier, and N. Bonnet, "Mechatronic design of a new robot for force control in minimally invasive surgery," IEEE/ASME Trans. Mechatron., vol. 12, no. 2, pp. 143-153, 2007.

[4] U. Hagn, T. Ortmaier, R. Konietschke, B. Kubler, U. Seibold, A. Tobergte, M. Nickl, S. Jorg, and G. Hirzinger, "Telemanipulator for remote minimally invasive surgery," IEEE Robot. Automat. Mag., vol. 15, no. 4, 2008.

[5] B. Rosa, C. Gruijthuijsen, B. Van Cleynenbreugel, J. Vander Sloten, D. Reynaerts, and E. Vander Poorten, "Estimation of optimal pivot point for remote center of motion alignment in surgery," International Journal of Computer Assisted Radiology and Surgery, vol. 10, no. 2, pp. 205$215,2015$.

[6] L. H. Clark, P. T. Soliman, D. Odetto, M. S. Munsell, K. M. Schmeler, N. Fleming, S. N. Westin, A. M. Nick, and P. T. Ramirez, "Incidence of trocar site herniation following robotic gynaecologic surgery," Gynecologic Oncology, vol. 131, no. 2, pp. 400-403, 2013.

[7] G. Scozzari, M. Zanini, F. Cravero, R. Passera, F. Rebecchi, and M. Morino, "High incidence of trocar site hernia after laparoscopic or robotic roux-en-y gastric bypass," Surgical Endoscopy, vol. 28, no. 10, pp. 2890-2898, 2014.

[8] R. Cortesao, W. Zarrad, P. Poignet, O. Company, and E. Dombre, "Haptic control design for robotic-assisted minimally invasive surgery," in 2006 IEEE International Conference on Intelligent Robots and Systems, 2006, pp. 454-459.
[9] A. Tobergte, R. Konietschke, and G. Hirzinger, "Planning and control of a teleoperation system for research in minimally invasive robotic surgery," in 2009 IEEE International Conference on Robotics and Automation, 2009, pp. 4225-4232.

[10] M. M. Dalvand and B. Shirinzadeh, "Remote centre-of-motion control algorithms of 6-rrcrr parallel robot assisted surgery system (pramiss)," in 2012 IEEE International Conference on Robotics and Automation, 2012, pp. 3401-3406.

[11] N. Aghakhani, M. Geravand, N. Shahriari, M. Vendittelli, and G. Oriolo, "Task control with remote center of motion constraint for minimally invasive robotic surgery," in 2013 IEEE International Conference on Robotics and Automation, 2013, pp. 5807-5812.

[12] A. Krupa, G. Morel, and M. de Mathelin, "Achieving high-precision laparoscopic manipulation through adaptive force control," Advanced Robotics, vol. 18, no. 9, pp. 905-926, 2004.

[13] C. Doignon, F. Nageotte, and M. de Mathelin, "The role of insertion points in the detection and positioning of instruments in laparoscopy for robotic tasks," in 2006 Medical Image Computing and ComputerAssisted Intervention, R. Larsen, M. Nielsen, and J. Sporring, Eds., 2006, pp. 527-534.

[14] S. Voros, J.-A. Long, and P. Cinquin, "Automatic detection of instruments in laparoscopic images: A first step towards high-level command of robotic endoscopic holders." The International Journal of Robotics Research, 2007.

[15] A. Krupa, J. Gangloff, C. Doignon, M. F. de Mathelin, G. Morel, J. Leroy, L. Soler, and J. Marescaux, "Autonomous 3-d positioning of surgical instruments in robotized laparoscopic surgery using visual servoing," IEEE Trans. Robot. Automat., vol. 19, no. 5, pp. 842-853, 2003.

[16] F. Nageotte, L. Ott, P. Zanne, and M. de Mathelin, "Analysis and improvement of image-based insertion point estimation for robot-assisted minimally invasive surgery," in 2009 IEEE International Conference on Robotics and Automation, 2009, pp. 3470-3475.

[17] J. T. Wilson, T. C. Tsao, J. P. Hubschman, and S. Schwartz, "Evaluating remote centers of motion for minimally invasive surgical robots by computer vision," in 2010 IEEE/ASME International Conference on Advanced Intelligent Mechatronics, 2010, pp. 1413-1418.

[18] E. Coste-Manière, L. Adhami, F. Mourgues, and O. Bantiche, "Optimal planning of robotically assisted heart surgery: First results on the transfer of precision in the operating room," The International Journal of Robotics Research, vol. 23, no. 4-5, pp. 539-548, 2004

[19] E. Ruiz Morales and C. Correcher Salvador, "Force estimation for a minimally invasive robotic surgery system," U.S. Patent US 2010/0 094312 A1, 2010.

[20] C. J. P. del Pulgar and V. F. Muoz, "Control scheme with tissue interaction detection for a single port access surgery robotic platform," in 2014 IEEE RAS/EMBS International Conference on Biomedical Robotics and Biomechatronics, 2014, pp. 713-718.

[21] A. Javaux, L. Esteveny, D. Bouget, C. Gruijthuijsen, D. Stoyanov, T. Vercauteren, S. Ourselin, D. Reynaerts, K. Denis, J. Deprest, and E. V. Poorten, "Body wall force sensor for simulated minimally invasive surgery: Application to fetal surgery," in 2017 IEEE/RSJ International Conference on Intelligent Robots and Systems, 2017, pp. 145-152.

[22] T. Ortmaier and G. Hirzinger, "Cartesian control issues for minimally invasive robot surgery," in 2000 IEEE/RSJ International Conference on Intelligent Robots and Systems, vol. 1, 2000, pp. 565-571 vol.1.

[23] L. Dong and G. Morel, "Robust trocar detection and localization during robot-assisted endoscopic surgery," in 2016 IEEE International Conference on Robotics and Automation, 2016, pp. 4109-4114.

[24] S. Thrun, W. Burgard, and D. Fox, Probabilistic Robotics. The MIT Press, 2005.

[25] I. Nisky, F. Huang, A. Milstein, C. Pugh, F. Mussa-Ivaldi, and A. Karniel, "Perception of stiffness in laparoscopy: the fulcrum effect," Studies in health technology and informatics, vol. 173, pp. 313-319, 2012.

[26] M. Michelin, P. Poignet, and E. Dombre, "Dynamic task/posture decoupling for minimally invasive surgery motions," in International Symposium on Experimental Robotics, 2007. 